\title{
Quelques réflexions sur les hydrocyclones en séparation liquide-liquide
}

PAR

P. Minart

Ingénieur E.E.G.

Alsthom T.D.F.

\section{Introduction}

Les hydrocyclones sont principalement utilisés dans le traitement des suspensions liquide-solide. Leur emploi dans le traitement des émulsions, en vue de la séparation des deux phases liquides, est beaucoup moins répandu.

Et pourtant, les hydrocyclones présentent des avantages par rapport aux autres appareils séparateurs habituellement utilisés, tels que les décanteurs et les centrifugeuses. Ils sont beaucoup plus compacts que les décanteurs. Ils sont dépour. vus de pièces mobiles, à la différence des centrifugeuses. Or l'on sait que dans ces machines, les pièces mobiles entraînent des servitudes de construction, de fonctionnement et d'entretien.

Nous nous proposons ci-après, d'examiner les particularités de fonctionnement d'un hydrocyclone traitant une émulsion. Nous verrons que, selon les conditions d'emploi, l'hydrocyclone peut fonctionner en séparateur ou en mélangeur. Ce comportement particulier explique, sans doute, pourquoi l'hydrocyclone n'est pratiquement pas utilisé industriellement pour la séparation liquide-liquide.

Nous verrons enfin comment, en tenant compte des servitudes de fonctionnement de l'hydrocyclone, nous avons pu étudier et réaliser un appareil séparateur qui a donné toute satisfaction en extraction liquide-liquide, à l'échelle pilote, pour des débits compris entre 30 et $300 \mathrm{l} / \mathrm{h}$.

\section{Rappel des propriétés des émulsions}

Les émulsions sont des dispersions plus ou moins stables de deux phases liquides non miscibles. La stabilité de l'émulsion dépend de la taille des gouttelettes de la phase dispersée, de l'intensité de la tension interfaciale, de la différence de densité des deux phases.

Les forces de surface liées à la dimension des gouttelettes et parfois renforcées par la présence, à l'interface, d'un agent émulsifiant tensio-actif, s'opposent à l'action de la pesanteur. Il est donc parfois nécessaire, avant d'envisager la séparation des deux phases, de procéder au préalable à un traitement destiné à détruire la stabilité de l'émulsion. Les procédés employés pour «casser » une émulsion sortent du cadre de cet exposé. Celui-ci est limité au traitement d'une émulsion instable où les phases de densités différentes tendent naturellement à se séparer sous l'action de la pesanteur.

\section{Etude documentaire et critique}

L'hydrocyclone, par la mise en œuvre d'accélérations centrifuges, permet d'accroître la vitesse de séparation des deux phases. On trouve, dans la littérature, un certain nombre d'études sur le sujet, peu nombreuses en vérité comparées à celles consacrées aux cyclones utilisés pour les séparations solides-gaz (cyclones dépoussiéreurs) et solidesliquides (hydrocyclones).

Les auteurs décrivent les essais qu'ils ont effectués avec des hydrocyclones de forme classique, à partir de dispersions liquide/liquide variées, huile/eau, tétrachlorure de carbone/eau, kérosène/eau, etc.

La séparation de deux liquides non miscibles dans un hydrocyclone est un problème complexe, étant donné le grand nombre de paramètres susceptibles d'intervenir :

- géométrie du cyclone;

- propriétés physiques des phases à séparer, telles que densité, viscosité, tension superficielle; 
- conditions d'alimentation, de fonctionnement et de soutirage des deux débits de sortie.

Il n'est donc pas étonnant de constater qu'il n'existe pas, à notre connaissance, une étude exhaustive de la question.

Les auteurs ont observé que la séparation des phases dans un hydrocyclone n'était que partielle et ils ont étudié l'influence des paramètres suivants sur l'efficacité de la séparation :

\section{La distribution granulométrique des gouttelettes à l'entrée [1]}

L'efficacité de la séparation augmente avec la taille des gouttelettes à l'entrée, comme il fallait s'y attendre. Cependant, à la différence des suspensions, la répartition granulométrique n'est pas un facteur clef.

En effet, cette répartition n'est pas stable dans le temps et dépend des conditions d'écoulement. Il peut y avoir coalescence, ou au contraire cisaillement des gouttelettes.

Finalement, il est impossible de définir un diamètre de coupure D 50, comme on le fait pour les séparations solide/gaz, solide/liquide.

\section{La concentration volumique de la phase dispersée à l'entrée [1][2]}

L'efficacité de séparation, toutes choses égales par ailleurs, est meilleure lorsque la concentration de la phase dispersée est plus faible. Ceci peut s'expliquer par l'influence de la viscosité [3], la force de traînée étant proportionnelle à la viscosité, l'efficacité de la séparation diminue lorsque la viscosité augmente.

Or l'on sait que la viscosité d'une émulsion augmente généralement avec la concentration de la phase dispersée et passe par un maximum au voisinage du point d'inversion [4].

\section{La répartition des débits de sortie de l'hydrocylone [1][2][3]}

La plupart des expérimentateurs ont fait varier la répartition des débits de sortie, soutirés respectivement à la surverse et à la sousverse.

Il apparaît que, pour obtenir une surverse pure ou aussi pure que possible, il faut opérer avec un faible débit de surverse. Il en est de même pour la sousverse.

Bohnet [2], par exemple, à partir d'un mélange huile-eau à $36 \%$ d'eau ne peut obtenir une huile pure à la surverse que si le rapport des débits surverse/sousverse est inférieur à 0,1 . Corollairement, pour obtenir de l'eau pure à la sousverse, le rapport précédent doit être supérieur à 10 .

Pour obtenir deux phases pures, Bohnet, comme Hitchon [5], propose d'opérer avec deux hydrocyclones en série (fig. 1). Le premier fournit la phase lourde, le second, traitant la surverse du premier, fournit la phase légère. La sousverse du second hydrocyclone est recyclée à l'amont du premier.

L'inconvénient de ce système, c'est que le débit recyclé est très important, si bien que le débit réellement traité est sept à huit fois plus élevé que le débit d'alimentation.

Rossum [3], quant à lui, observe qualitativement le même phénomène mais ne parvient pas à réduire la teneur en eau de la surverse au-dessous de $0,5 \%$. Il en conclut qu'il se produit dans l'hydrocyclone un mélange indésirable.

\section{L'influence de la vitesse d'entrée}

La plupart des expérimentateurs n'ont pas étudié l'influence de ce paramètre. Rossum, cependant, constate que l'efficacité de séparation n'est pas meilleure lorsque la vitesse d'entrée passe de 8 à $16 \mathrm{~m} / \mathrm{s}$. Il explique ce résultat par le fait que l'augmentation de vitesse a deux conséquences opposées.

D'une part, la force centrifuge est accrue, mais, d'autre part, les forces de cisaillement le sont également, si bien qu'il en résulte une pulvérisation des gouttelettes. Bohnet fait varier la vitesse dans de grandes proportions et met ainsi en évidence une vitesse critique optimale pour la séparation, de l'ordre de 3 à $4 \mathrm{~m} / \mathrm{s}$.

Aux vitesses inférieures à la vitesse critique, l'hydrocyclone fonctionne en séparateur, tandis qu'aux vitesses supérieures il fonctionne en émulseur.

La vitesse critique est indépendante de la répartition des débits à la sortic, par contre, elle varie avec la concentration de la phase dispersée à l'entrée.

Ces résultats expérimentaux sont très importants, car ils mettent en évidence les particularités de fonctionnement de l'hydrocyclone en séparation liquide-liquide.

Contrairement à ce qui se passe en séparation liquidesolide, la turbulence de l'écoulement en séparation liquideliquide joue un rôle très important voire prépondérant.

Une turbulence modérée joue un rôle favorable, car elle augmente la probabilité de rencontre et de collision des gouttelettes, favorisant ainsi leur coalescence. Le mécanisme est analogue à celui que l'on observe lors de la floculation orthocinétique.

Une turbulence intense, au contraire, est défavorable, car elle provoque le cisaillement des gouttelettes, analogue à la «poussiérisation» des flocs en floculation.

Le lait fournit un exemple des effets contraires de l'agitation selon son intensité : on peut l'homogénéiser en l'agitant énergiquement pendant un temps très court. La crèms, émulsion laiteuse concentrée, peut être cassée avec formation de beurre par barattage lent pendant un temps prolongé

\section{Influence de la tension interfaciale}

Il est connu que l'énergie mécanique nécessaire pour former une émulsion est d'autant plus faible que la tension interfaciale est plus petite. On voit donc que, dans un hydrocyclone, la turbulence, et donc la vitesse optimale d'entrée, dépendent de la tension interfaciale.

\section{Etude expérimentale}

Lorsqu'en 1960, nous avons entrepris nos études en vue de la réalisation d'un hydrocyclone séparateur utilisable en extraction liquide-liquide, la plupart des travaux que nous venons d'analyser n'étaient pas encore publiés.

Nous avons constaté le comportement particulier de l'hydrocyclone qui, selon la vitesse d'entrée, fonctionnait en séparateur ou en mélangeur. Plus précisément, il apparut que l'efficacité de séparation était maximale pour une valeur déterminée de la vitesse d'entrée. 
En outre, les essais ont conduit aux conclusions suivantes :

- L'hydrocyclone de forme classique n'est pas approprié, puisqu'il ne permet pas d'obtenir simultanément les deux débits de sortie suffisamment purifiés.

La combinaison de deux cyclones en série, proposée par Hitchon, ne parait pas la bonne solution, puisquelle conduit, par suite du recyclage, à des vitesses d'entrée prohibitives que, précisément, il faut éviter.

Nous nous sommes orientés vers un hydrocyclone modifié, capable de traiter un débit maximum avec une assez bonne efficacité de séparation.

- Le débit maximum dépend de la concentration de la phase dispersée. Il dépend aussi de la nature de la phase dispersée. Partant d'un mélange white spirit/eau à $30 \%$ d'eau, on obtient une phase aqueuse pure et une phase légère contenant seulement 1 à $2 \%$ d'eau.

Pour obtenir les mêmes résultats, à partir d'un mélange essence de térébenthine/eau, il faut opérer, sur le même appareil, avec un débit beaucoup plus faible. Ainsi le volume et la géométrie de l'appareil doivent être adaptés à la nature de la dispersion et au débit à traiter.

- Les paramètres géométriques importants sont, outre la section d'alimentation tangentielle qui fixe la vitesse d'entrée, la section de sortie de la surverse, le volume de l'hydrocyclone.

La section de sortie de la surverse dépend du débit de phase légère.

Le volume de l'hydrocyclone doit être déterminé pour avoir un temps de séjour suffisant de l'ordre de cinq secondes.

- La séparation des phases au sein du cyclone ne suffit pas. Encore faut-il obtenir une répartition correcte des débits de sortie correspondant à la composition du débit d'alimentation.

Pour assurer cette répartition, il est nécessaire et il suffit de réguler un débit de sortie, le débit le plus faible de préférence, c'est-à-dire le débit de phase dispersée.

\section{Description des appareils réalisés}

Compte tenu de ce qui précède, les séparateurs que nous avons réalisés [7] comprennent essentiellement trois parties:

- une partie médiane qui est l'hydrocyclone proprement dit;

- une chambre de régulation du débit de phase dispersée; - une chambre de finition de la phase continue.

\section{Séparateur à phase légère continue} (fig. 2)

Nous nous limiterons ci-après à la description détaillée d'un séparateur destiné à traiter une dispersion liquideliquide où la phase légère est la phase continue à débit prépondérant.

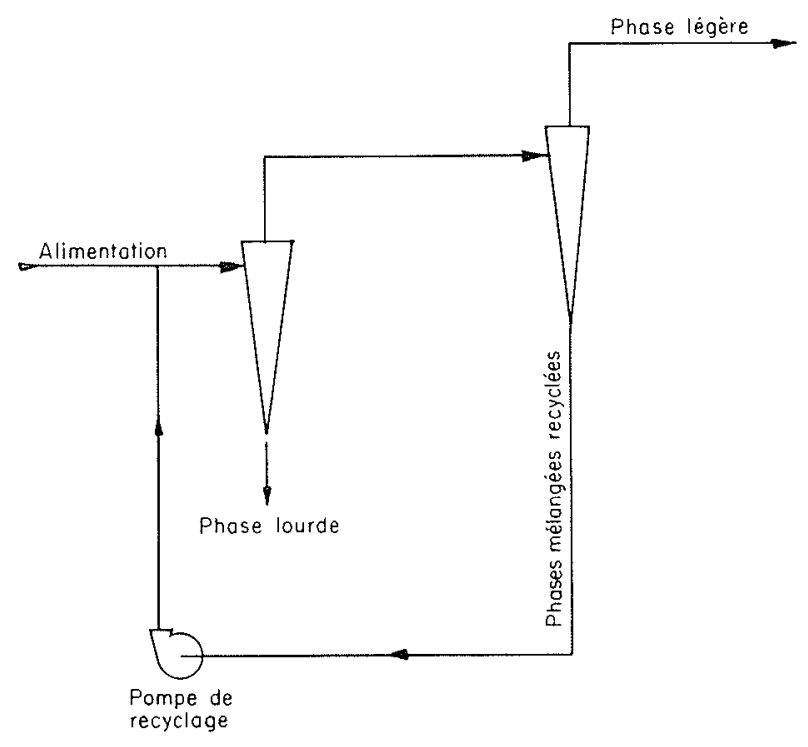

$1 /$ Séparation liquide-liquide par deux hydrocyclones en série.

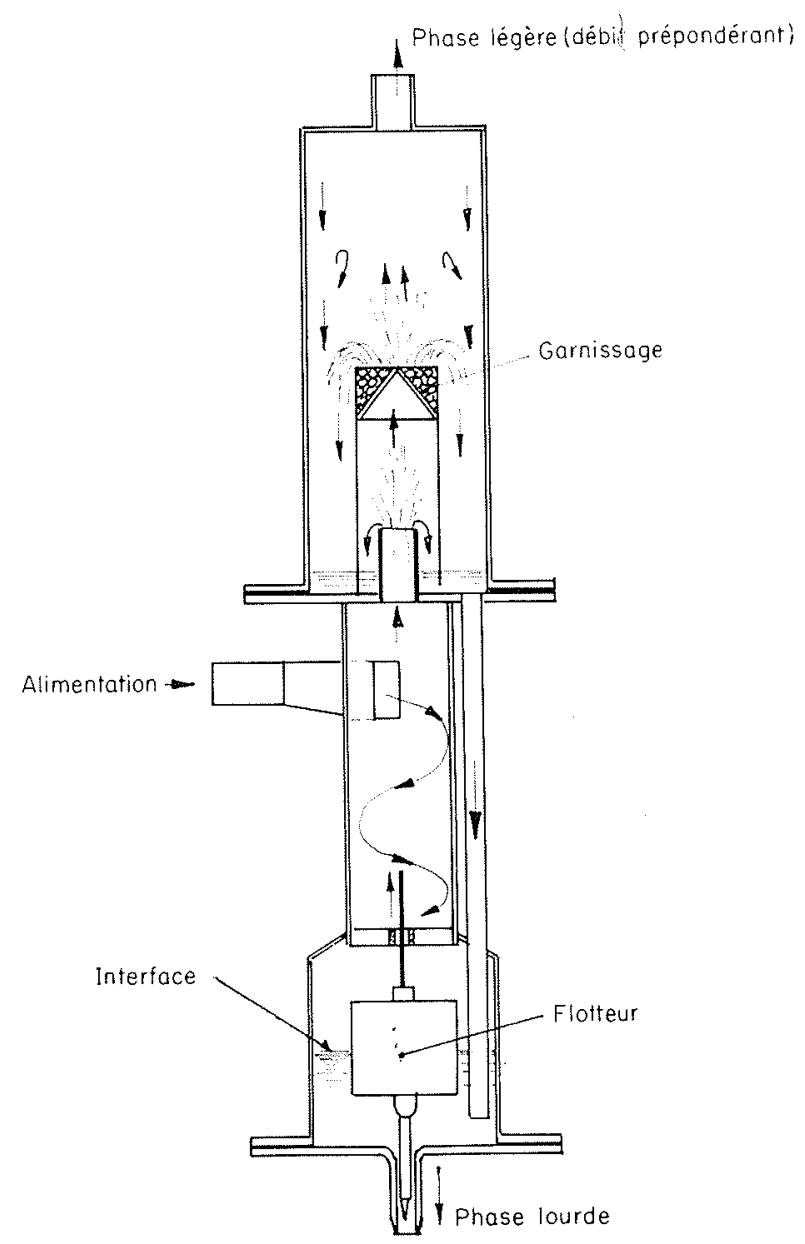

2/ Séparateur à phase légère continue. 


\section{L'hydrocyclone}

Il est de forme cylindrique et non pas conique, afin de permettre une diminution progressive de la vitesse de haut en bas, comme nous le verrons au paragraphe suivant.

Le rapport hauteur/diamètre est important, afin d'avoir, d'une part, une accélération centrifuge suffisante et, d'autre part, un temps de séjour approprié. Les gouttelettes lourdes dispersées sont projetées sur les parois sous l'action de la force centrifuge. Elles coaleseent dans la couche limite et, sous laction de la pesanteur, s'écoulent vers le bas en suivant des trajectoires hélicoïdales.

\section{La chambre de régulation de débit}

Dans la chambre inféricure, il se forme une interface phase lourde, phase légère. On profite de la présence de cette interface pour réguler le débit de sortie de la phase lourde au moyen d'un flotteur taré qui flotte à l'interface. Le flottcur est muni d'une tige inférieure de guidage qui pénètre dans l'orifice de sortie, à la manière d'un pointeau. Le flotteur suit les déplacements de linterface et obture plus ou moins lorifice de sortie. Il assure ainsi, par variation de perte de charge, la régulation automatique du débit de sortie. Afin d'éviter que le flotteur ne soit sollicité par des forces autres que la poussée d'Archimède, un certain nombre de précátutions sont prises. La section de la chambre est telle que les forces de traînée agissant sur le flottcur soient négligeables. Par ailleurs, la vitesse de rotation du liquide est annulée par un croisillon profilé, placé entre l'hydrocyclone et la chambre de régulation de débit.

\section{La chambre de finition de la phase continue}

Comme on l'a vu précédemment, s'il est possible à l'aide d'un hydrocyclone d'obtenir de la phase dispersée pratiquement pure, la purification de la phase continue n'est jamais complète. Nous avons donc équipé nos appareils d'une chambre de finition destinée à parfaire l'épuration de la phase continue.

Les principes physiques mis en auvre sont les suivants: - l'effet de jet,

- la coalescence des micro-gouttelettes par filtration sur un garnissage.

La phase légère, sortant de l'hydrocyclone par l'orifice de surverse, forme un jet dans la chambre de finition. II se produit, à la périphérie du jet, des courants de recirculation qui favorisent la sédimentation des gouttelettes lourdes entrainées.

Le jet s'écoule ensuite à travers un garnissage mouillé préférentiellement par la phase continue, qui retient la plupart des fines gouttelettes et provoque leur coalescence. On a essayé également un garnissage mouillé préférentiellement par la phase dispersée, mais les essais n'ont pas été concluants.

Le garnissage repose sur un cône perforé, supporté à une hauteur suffisante par un tube concentrique échancré à la base.

Lorsque le jet atteint le garnissage, au lieu d'être étalé et redispersé, comme ce serait le cas si l'on avait un garnissage d'épaisseur uniforme, il reste centré sur l'axe où la perte de charge est moindre.
Les micro-gouttelettes coalescent dans le garnissage. Il se forme, à la partie supérieure de ce dernier, de grosses gouttes de phase lourde, qui, lorsque leur taille est suffisante, s'écoulent d'elles-mêmes vers le bas de la chambre, dans lespace annulaire compris entre le cylindre support et la paroi.

L'évacuation des gouttes coalescées, par l'aval du garnissage, évite leur redispersion dans le courant principal, telle qu'elle peut se produire lorsque la vidange du garnissage s'effectue par l'amont. Le garnissage ne provoque pas l'arrêt total des gouttelettes de phase lourde. Mais, au-dessus du garnissage, un nouvel effet de jet se produit avec sédimen. tation des gouttelettes le long des parois. La phase lourde collectée à la base de la chambre de finition s'écoule dans la chambre inférieure par un tube vertical de liaison.

Grâce à la chambre de finition, nous avons pu limiter l'entraînement dans la phase solvant à moins de 0,5 à $1 \%$ selon les cas, avec un seul appareil et à moins de $0,1 \%$ avec deux appareils en série.

Signalons enfin que, lorsque l'on traite une dispersion très fine, on peut augmenter la taille des gouttelettes à l'entrée en plaçant, à l'amont du cyclone, un appareil précoalesceur très simple [8].

\section{Séparateur à phase lourde continue} (fig. 3)

L'appareil est analogue au précédent, mais la position respective des deux chambres de régulation et de finition est inversée. L'hydrocyclone comprend une partie conique destinée à ramener, sur l'axe, l'écoulement de phase lourde avant son entrée dans la chambre de finition.

\section{Conclusion}

Il est possible de séparer les deux phases liquides non miscibles d'une émulsion déstabilisée au moven d'un hydrocyclone.

La séparation est facilitée, par une différence de densité et une tension interfaciale élevées, par une viscosité faible de la phase continue et de l'émulsion, par une faible concentration de la phase dispersée.

Lorsque l'on a à traiter une émulsion particulièrement fine, comme c'est le cas par exemple en extraction liquideliquide, on peut accroître la taille des micro-gouttelettes à l'aide d'un dispositif simple placé à l'amont de l'appareil.

Le volume et la géométrie de l'hydrocyclone doivent être adaptés à la nature de la dispersion et au débit à traiter. En particulier, la vitesse d'entrée doit être ajustée à la valeur optimale pour éviter que lappareil ne fonctionne en émulseur. Dans ces conditions, il est relativement aisé d'obtenir de la phase dispersée à l'état pur. Par contre, la phase continue exige un traitement de finition. On peut, soit utiliser un second hydrocyclone en série avec le premier, soit équiper l'appareil d'une chambre de finition.

La régulation du débit de sortie le plus faible (phase dispersée) permet d'éviter l'emploi de deux appareils en 


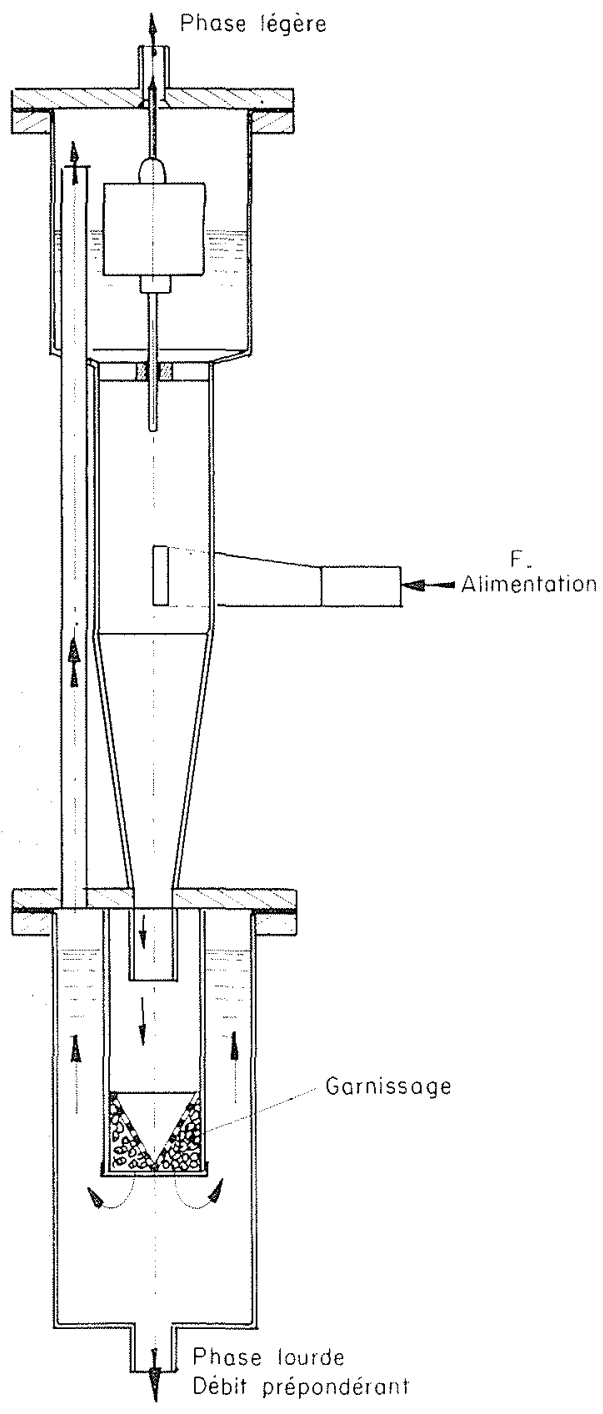

3/ Séparateur à phase lourde continue.

série avec recyclage du débit non traité à l'amont du premier cyclone.

Un tel appareil a été utilisé avec succès dans une installation pilote d'extraction liquide-liquide à trois étages; chaque étage comprenant un éjecteur-mélangeur et un séparateur cyclone.

\section{Références}

[1] BuRrtl, (K. A.) and Woons (D. R.). - Ind, Eng. Chem., Vol. 9, $\mathrm{n}^{*} 4,545(1970)$.

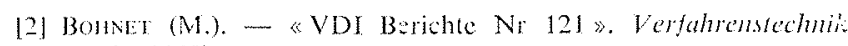
153 (1968).

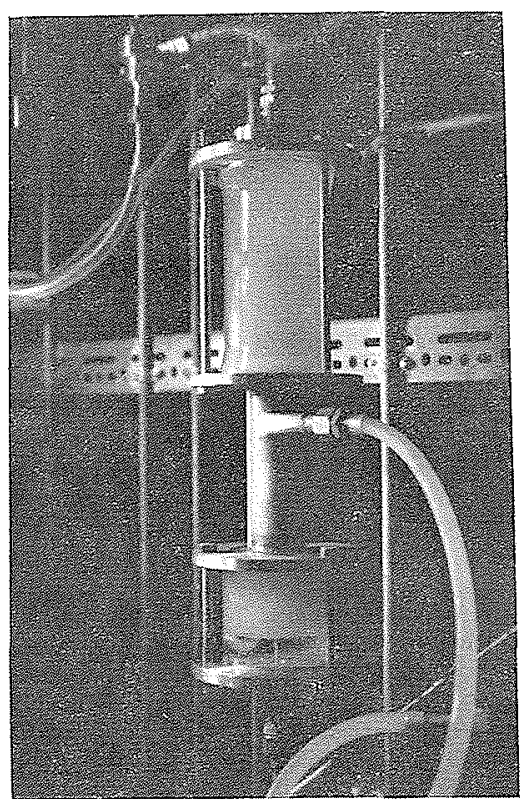

extraction

liquide-liquide.

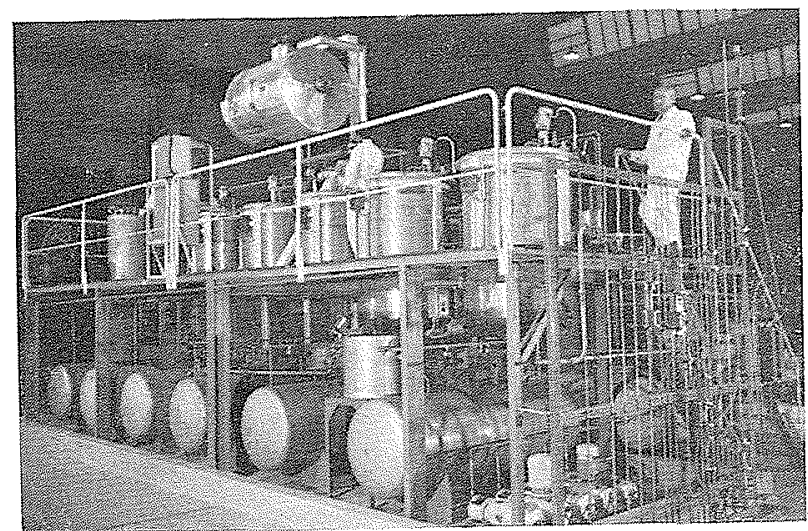

5. Cascade à trois étages éjecteurs-hydrocyclones pour l'extraction liquide-liquide (à droile de la photo).

13] Vas Rossum (J.J.) in s Cyclones in - Industry $\$$ Rietema of Verver, p. 110 (1961).

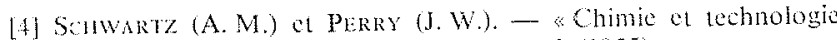
des agents tensiomactifs » (355). Dumod (1955).

15 Hrichos (J. W.). - Cyclones as liquid-liquid contactorsseparators. AERE-CE/R 2777 (1959).

16] Kindrg (J.K.) and HAZEN (W.C.). - Transaction of the socicly of Mining engincers. AlME, Vol. 250 (1971).

[7] [8] Faugeras (P.), Minart (P.), Pouchot (P.), Talmont (X.): - Apparcil pour la séparation de deux phases liquides non miscibles. $\gg$ Brevet $\mathrm{n}^{\prime \prime} 1297313$ (délivré le 21 mai 1962). - Procédé pour lamélioration du rendement de séparation des cyclones utilisés en extraction liquide-fiquide. $\gg$ Brevei n" 1328511 (mai 1963).

- Installation pour l'extraction liquide-liquide à contro courant. B Brevet 11" 1328538 (avril 1962). 


\section{Discussion}

Président: M. le Professeur A. Fontuer

M. le Président remercie M. Minart de son fort intéressant exposé et ouvre la discussion.

Sur une question de M. Martin, M. Minart précise:

Nos études et nos installations ont été faites à l'échelle pilote stir du nitrate d'uranyle en présence d'un solvant tributylphosphate pour des débits de $300 \mathrm{l} / \mathrm{h}$; les résultats ont été excellents. En trois étages en cascade, on arrivait à faire passer la concentration en uranium de $300 \mathrm{~g} / 1$ à 10 ou $20 \mathrm{mg} / \mathrm{l}$, ce qui correspond à trois étages théoriques

Le problème qui se pose évidemment est celui de l'extrapolatio: à pius grande échelle. Je dois dire que nous ne connaissons pas de règles précises dextrapolation; compte tenu du nombre de paramé tres qui entrent en jeu, on se heurte à un certain nombre de difficultés. Pour des appareils de grande puissance, il faudrait passer par une étape intermédiaire de dimension supérieure.

Notre installation a été réalisée à partir d'une installation traitant du white spirit et de l'eau, dont le débit était dix fois plus faible. Les règles d'extrapolation, que nous avons utilisées pour passer de 301 ì $300 \mathrm{l} / \mathrm{h}$, se sont révélées suffisamment bonnes pour que les résultats du traitement soient satisfaisants et comparables.

A theure actuelle, nous utilisons et nous développons un appareil que nous appelons le «Cyclonet» qui sert à l'écrémage du fuel ou du mazout sur la mer ou dans les ports. C'est beaucoup plus un écrémeur qu’un séparateur puisqu'jl est utilisé dans le cas où Io fuel se trouve déjà rassemblé en surface.

Pour la mise au point du «Cyclonet», nous avons utilisé certain résultats obtenus il y a quinze ans dans nos études précitées sur la séparation liquide-tiquide.

A-t-on des informations plus précises, demande $M$. le Président, sur l'influence de la turbulence et par conséquent, des dimensions de l'appareil sur son fonctionnement. En fait, la turbulence fait interveni deux paramètres: une échelle de longueur - liée aux dimensions de l'appareil - et une échelle de temps (liée au rapport longueur/ vitesse).

Dans votre dispositif, vous avez indiqué des vitesses optimales de 3 a $4 \mathrm{~m} / \mathrm{s}$. Quelles devraient être les valeurs de ces vitesses si l'on change les dimensions de lappareil?

Nous n'avons pas fait d'études suffisantes en matière d'extrapolation de notre dispositif, répond $M$. Mrnart.

Pensez-vous, demande M. Martin, que vos essais de séparation du white-spirit et de l'eau conduisent à des résultats économiquement pius avantageux quine séparation par distillation, interroge M. MARTIN?

Je me suis servi du white-spirit et de lessence de thérébentine au départ, rópond $M$. MinarT, pour dégrossir le problème et me rendre compte de linfluence des tensions interfaciales. Mais je n'ai pas envisagé le problème sous Jangle économique.

Mon but était de comparer la cascade «éjecteur-cyclone» avec les mélangeurs-décanteurs et les colonnes pulsées, susceptibles d'être utilisés pour ie mème objet.

Je me demandais, ajoute $M$. MarTiN, si le procédé qui nous a étó décrit pourrait s'appliquer à la séparation d'une émulsion d'eau ct de white-spirit (30 a $40 \%$ en phase dispersée) après son utilisation pour le dégraissage des peaux (peaux de mouton, notamment). La séparation par distillation est réalisable mais jugée trop onéreuse.

M. Minart le pense, car ses essais ont aussi porté sur l'apparei? de la figure 3 de sa communication, en utilisant une émulsion où leau était prépondérante et constituait la phase continue.

En labsence d'autre intervention, M. le Président lève la séance à $12 \mathrm{~h} 15$ en remerciant tous ceux qui ont contribué à en soutenir lintérêt. 